\title{
Exact Solution of the Spin-Isospin Proton-Neutron Pairing Hamiltonian
}

\author{
S. Lerma H., ${ }^{1}$ B. Errea, ${ }^{1}$ J. Dukelsky, ${ }^{1}$ and W. Satuła ${ }^{2}$ \\ ${ }^{1}$ Instituto de Estructura de la Materia, CSIC, Serrano 123, 28006 Madrid, Spain \\ ${ }^{2}$ Institute of Theoretical Physics, University of Warsaw, ul. Hoza 69, 00-681 Warsaw, Poland
}

(Received 20 March 2007; published 17 July 2007)

\begin{abstract}
The exact solution of the proton-neutron isoscalar-isovector $(T=0,1)$ pairing Hamiltonian with nondegenerate single-particle orbits and equal pairing strengths is presented for the first time. The Hamiltonian is a particular case of a family of integrable SO(8) Richardson-Gaudin models. The exact solution of the $T=0,1$ pairing Hamiltonian is reduced to a problem of 4 sets of coupled nonlinear equations that determine the spectral parameters of the complete set of eigenstates. The microscopic structure of individual eigenstates is analyzed in terms of evolution of the spectral parameters in the complex plane for a system of $A=80$ nucleons. The spectroscopic trends of the exact solutions are discussed in terms of generalized rotations in isospace.
\end{abstract}

DOI: 10.1103/PhysRevLett.99.032501

The exactly solvable models introduced by Richardson [1] and by Gaudin [2] belong nowadays to classic theoretical tools in mesoscopic physics. Indeed, these models based on the rank $1 \mathrm{SU}(2)$ algebra for fermions or the $\mathrm{SU}(1,1)$ algebra for bosons were applied to a large variety of quantum many-body systems including the atomic nucleus, superconducting grains, cold atomic gases, etc.; see review article [3] and references therein. Recently, we have extended the Richardson-Gaudin (RG) models to the rank 2 algebras: $\mathrm{SO}(5)$ (isovector pairing [4]), $\mathrm{SO}(3,2)$ ( $F$ spin 1 boson pairing [5]), and $\mathrm{SU}(3)$ (interacting three level atoms [6]).

In this Letter we will derive for the first time the exact solution for the rank $4 \mathrm{SO}(8) \mathrm{RG}$ integrable model with nondegenerate single-particle $(s p)$ spectrum and arbitrary degeneracies. As a particular realization of the rank 4 $\mathrm{SO}(8) \mathrm{RG}$ model we will consider the nuclear isoscalarisovector $(T=0,1)$ pairing Hamiltonian introduced for a single degenerate shell in Ref. [7] and further developed in $[8,9]$. It should be mentioned that other representations like the Ginnocchio model [10] can lead to interesting exactly solvable models in nuclear structure as well as to models of spin $3 / 2$ cold atoms [11].

The study of proton-neutron $(p-n)$ pairing has gained a renewed interest due to the new generation of radioactivebeam facilities that will open the access to proton-rich nuclei close to the $N=Z$ line. In spite of vigorous activity in this field, see [12] and references therein, the fundamental questions concerning the basic building blocks and experimental fingerprints of the $p-n$ pairing are still a matter of debate. So are the theoretical problems concerning generalizations of well established nuclear pairing models to include $p-n$ pairing, proper treatment of the isospin degree of freedom, or $\alpha$-like clustering and quartet condensation [13]. All these problems set clear motivations for realistic exact-model studies of the $p-n$ pairing undertaken in this work.

Let us begin our derivation by introducing the 28 generators of the $\mathrm{SO}(8)$ algebra [7]: three $(T=1$,
PACS numbers: 21.60.Fw, 02.30.Ik, 74.20.Rp

$S=0)$ and three $(T=0, S=1)$ pair creators, together with their respective annihilation operators: $\mathbf{P}_{\tau i}^{\dagger}=$ $\sqrt{\left(2 l_{i}+1\right) / 2}\left[\mathbf{a}_{l_{i}}^{\dagger} \mathbf{a}_{l_{i}}^{\dagger}\right]_{0 \tau 0}^{010}, \quad \mathbf{D}_{\sigma i}^{\dagger}=\sqrt{\left(2 l_{i}+1\right) / 2}\left[\mathbf{a}_{l_{i}}^{\dagger} \mathbf{a}_{l_{i}}^{\dagger}\right]_{00 \sigma}^{001}$, $\mathbf{P}_{\tau i}=\left(\mathbf{P}_{\tau i}^{\dagger}\right)^{\dagger}$, and $\mathbf{D}_{\sigma i}=\left(\mathbf{D}_{\sigma i}^{\dagger}\right)^{\dagger}$, where the triads in the couplings represent, respectively, angular momentum, isospin, and spin. The fermionic operators $\mathbf{a}_{l_{i} m \tau \sigma}^{\dagger}$ create a particle in the orbit $l_{i}$ with projection $m$, isospin $\tau$, and spin $\sigma$. The $\mathrm{SO}(8)$ algebra is completed by the 16 particlehole operators: $\mathbf{C}_{\tau_{1} \sigma_{1}, \tau_{2} \sigma_{2} ; i} \equiv \sum_{m} \mathbf{a}_{l_{i} m \tau_{1} \sigma_{1}}^{\dagger} \mathbf{a}_{l_{i} m \tau_{2} \sigma_{2}}$. These 16 operators close a $\mathrm{U}(4)$ subalgebra of $\mathrm{SO}(8)$.

The formal solution of the Gaudin-type models for arbitrary simple Lie algebras has been developed in Refs. $[14,15]$. For each algebra it is possible to derive a set of $L$ quadratic integrals of motion defining the integrable model. It is also possible to derive the complete set of common eigenstates and eigenvalues, which constitute the exact solution of the model. Here $L$ is the number of copies of the algebra that we associate with the number of orbits $l_{i}$. For simplicity we will consider here a particular linear combination of the integrals of motion of the $\mathrm{SO}(8)$ RG model giving rise to the $T=0,1 \quad p-n$ pairing Hamiltonian of equal strengths $g_{T=0}=g_{T=1}=g$ :

$$
\mathbf{H}=\sum_{i}^{L} \epsilon_{i} \mathbf{N}_{i}-g \sum_{i i^{\prime}}^{L} \sum_{\mu}\left(\mathbf{P}_{\mu i}^{\dagger} \mathbf{P}_{\mu i^{\prime}}+\mathbf{D}_{\mu i}^{\dagger} \mathbf{D}_{\mu i^{\prime}}\right),
$$

where $\mathbf{N}_{i}$ is the number operator of the orbit $l_{i}$. The Hamiltonian (1) is rich enough to capture the essence of $p-n$ pairing and quarteting related phenomena. Depending on the situation, however, the model Hamiltonian can be extended by including isospin breaking terms and more general two-body interactions expressible in terms of general linear combinations of the integrals of motion.

A common strength $g$ implies a conservation of U(4) symmetry defined by the generators $\mathbf{C}_{\tau_{1} \sigma_{1}, \tau_{2} \sigma_{2}} \equiv$ $\sum_{i}^{L} \mathbf{C}_{\tau_{1} \sigma_{1}, \tau_{2} \sigma_{2} ; i}$. Therefore, the eigenstates are organized in degenerated U(4) Wigner multiplets. For a given number of nucleons $A$, these $U(4)$ multiplets can be classified 
using Young tableaux. Each multiplet is defined by a partition of $A$ in 4 numbers $\left[h_{1} h_{2} h_{3} h_{4}\right]$ constrained by $\sum_{i}\left(2 l_{i}+1\right) \geq h_{1} \geq h_{2} \geq h_{3} \geq h_{4} \geq 0$. The labels $h_{i}$ are related to the number of particles in the total U(4) lowest weight state (LWS). For instance, if we relabel the $\mathrm{U}(4)$ operators according to the rule $1 \equiv n \downarrow, 2 \equiv n \uparrow$, $3 \equiv p \downarrow$, and $4 \equiv p \uparrow$, the U(4) LWS can be defined as the state which satisfies $\mathbf{C}_{\alpha, \beta}|\mathrm{LWS}\rangle=0, \forall \alpha<\beta$. For this choice of LWS, the corresponding U(4) Young tableau is given by $\left[N_{n \downarrow} N_{n \uparrow} N_{p \downarrow} N_{p \uparrow}\right]$. The spin and isospin of this LWS are simply $2 S=N_{n \downarrow}+N_{p \downarrow}-N_{n \uparrow}-N_{p \uparrow}$ and $2 T=$ $N_{n \downarrow}+N_{n \uparrow}-N_{p \downarrow}-N_{p \uparrow}$.

As stated above, the eigenvalues of Hamiltonian (1) can be derived from the exact solution of the $\mathrm{SO}(8) \mathrm{RG}$ model. They are

$$
E=\sum_{\alpha}^{M_{1}} e_{\alpha}+\sum_{i=1}^{L} \epsilon_{i} u_{i}
$$

where $u_{i}$ is the seniority of level $i$, i.e., the number of particles in level $i$ not coupled in $T=1$ or $T=0$ pairs. The parameters $e_{\alpha}$ satisfy the generalized Richardson equations:

$$
\begin{aligned}
& \sum_{\alpha^{\prime}(=\alpha)}^{M_{1}} \frac{2}{e_{\alpha^{\prime}}-e_{\alpha}}- \sum_{\alpha^{\prime}}^{M_{2}} \frac{1}{\omega_{\alpha^{\prime}}-e_{\alpha}} \\
&-\sum_{i}^{L} \frac{\left(2 l_{i}+1\right)-h_{1 ; i}-h_{2 ; i}}{2 \epsilon_{i}-e_{\alpha}}+\frac{1}{g}=0, \\
& \sum_{\alpha^{\prime}(\neq \alpha)}^{M_{2}} \frac{2}{\omega_{\alpha^{\prime}}-\omega_{\alpha}}-\sum_{\alpha^{\prime}}^{M_{1}} \frac{1}{e_{\alpha^{\prime}}-\omega_{\alpha}}-\sum_{\alpha^{\prime}}^{M_{3}} \frac{1}{\eta_{\alpha^{\prime}}-\omega_{\alpha}} \\
&-\sum_{\alpha^{\prime}}^{M_{4}} \frac{1}{\gamma_{\alpha^{\prime}}-\omega_{\alpha}}+\sum_{i}^{L} \frac{h_{3 ; i}-h_{2 ; i}}{2 \epsilon_{i}-\omega_{\alpha}}=0, \\
& \sum_{\alpha^{\prime}(\neq \alpha)}^{M_{3}} \frac{2}{\eta_{\alpha^{\prime}}-\eta_{\alpha}}-\sum_{\alpha^{\prime}}^{M_{2}} \frac{1}{\omega_{\alpha^{\prime}}-\eta_{\alpha}}+\sum_{i}^{L} \frac{h_{4 ; i}-h_{3 ; i}}{2 \epsilon_{i}-\eta_{\alpha}}=0, \\
& \sum_{\alpha^{\prime}(\neq \alpha)}^{M_{4}} \frac{2}{\gamma_{\alpha^{\prime}}-\gamma_{\alpha}}-\sum_{\alpha^{\prime}}^{M_{2}} \frac{1}{\omega_{\alpha^{\prime}}-\gamma_{\alpha}}+\sum_{i}^{L} \frac{h_{2 ; i}-h_{1 ; i}}{2 \epsilon_{i}-\gamma_{\alpha}}=0,
\end{aligned}
$$

where $\left[h_{1 ; i} h_{2 ; i} h_{3 ; i} h_{4 ; i}\right]$ is the Young tableau of the reduced $\mathrm{U}(4)$ multiplet defined by the unpaired particles in the $i$ th orbit. These U(4) labels are constrained by the conditions $2 l_{i}+1 \geq h_{1 ; i} \geq h_{2 ; i} \geq h_{3 ; i} \geq h_{4 ; i} \geq 0$. In terms of these labels the seniority of level $i$ is $u_{i}=\sum_{k} h_{k ; i}$. The rank of the RG models defines the number of different sets of spectral parameters. $\mathrm{SO}(8)$ is a rank 4 algebra; hence, there are four sets of spectral parameters. The number of spectral parameters in each set is determined by the reduced labels and those of the total U(4) Wigner multiplet: $M_{1}=(A-$ u) $/ 2, M_{2}=h_{3}+h_{4}-\sum_{i}\left(h_{3 ; i}+h_{4 ; i}\right), M_{3}=h_{4}-\sum_{i} h_{4 ; i}$, and $M_{4}=\left(A-2 h_{1}\right) / 2-\left(u-\sum_{i} 2 h_{1 ; i}\right) / 2$, with $u=\sum_{i} u_{i}$.

The first set of spectral parameters comprises the usual pair energies $e_{\alpha}$ of the $\mathrm{SO}(8)$ algebra. The other three sets, composed by the spectral parameters $\omega_{\alpha}, \eta_{\alpha}$, and $\gamma_{\alpha}$, are associated with the $\mathrm{U}(4)$ subalgebra of $\mathrm{SO}(8)$. While the eigenvalues depend only on the parameters $e_{\alpha}$, the corresponding eigenfunctions are determined by the parameters of the four sets. The complete set of solutions of the Richardson equations defines a basis which spans completely the Hilbert space of sates with the same U(4) Wigner quantum numbers $\left[h_{1} h_{2} h_{3} h_{4}\right]$.

Even though the set of nonlinear coupled equations (3) seems to be extremely complex, we will show how it is possible to obtain numerical solutions within a nontrivial example of $A=4 n$ nucleons, with $n$ a positive integer, moving in a set of nondegenerate $l=0$ single-particle orbits. Other cases could be handled following a similar procedure. Before describing the numerical strategy, it will be useful to consider the lowest-energy LWS configurations for even and odd isospin in the $g=0$ limit.

For even $T$ the lowest $(A-2 T) / 4$ levels are filled with 4 nucleons, and the following $T$ levels with a pair of neutrons. All particles are paired and the corresponding seniority quantum numbers are 0 . In the case of $T$ odd, the levels $(A-2 T+2) / 4$ and $(A+2 T+2) / 4$ have one unpaired nucleon $\left(u_{(A-2 T+2) / 4}=u_{(A+2 T+2) / 4}=1\right)$. This state can be considered a $p$ - $h$ excitation that evolves to a twoquasiparticle state in the superconducting phase. As a consequence, the number of pair energies $e_{\alpha}$ is $M_{1}=$ $A / 2$ in the even $T$ case and $M_{1}=A / 2-1$ in the odd $T$ case. In this limit the pair energies take the values $e_{\alpha}=2 \epsilon_{i}$ according to the same pattern.

In the weak coupling limit $(g \ll 1)$ the Richardson equations (3) decouple into independent sets of equations, each one related to a single-particle level partially or fully occupied in the $g=0$ limit. These equations can be solved analytically. The 4 sets of spectral parameters obtained in this way are used as an initial guess for an iterative procedure in which the coupling constant $g$ is increased step by step using the previous solution as the initial the guess.

The main obstacle in solving the Richardson equations even in the $\mathrm{SU}(2)$ case is the appearance of singularities at some critical values of the pairing strength due to crossings in the real axis of single-particle energies and spectral parameters [16,17]. This problem is even worse in the $\mathrm{SO}(8)$ model having 4 sets of spectral parameters. In order to avoid these numerical instabilities we introduce an alternate imaginary term in the $s p$ energies $\epsilon_{i} \rightarrow \epsilon_{i}+$ $(-)^{i} \Delta \sqrt{-1}$, which breaks the time reversal symmetry and moves the solutions of (3) away from the real axis [18]. The system is then evolved from the initial guess at $g \ll 1$ to the desired value of $g$. At this point we begin a second iterative process to set the imaginary term in the single-particle energies to zero $(\Delta \rightarrow 0)$. This recursive procedure proved to be very efficient in solving the Richardson equations for arbitrary values of the coupling constant and for all the states considered.

We will now demonstrate the ability of our procedure for solving large scale complex physical problems by presenting a numerical example for a system of $A=80$ nucleons 
described by the Hamiltonian (1) and moving in a set of $L=50$ equidistant and fourfold degenerate levels $\left[\epsilon_{i}=\right.$ $(i-1) / 2, l_{i}=0$ with $\left.i=1, \ldots, 50\right]$.

Solving Eqs. (3) provides not only the exact spectroscopy but also allows for a pictorial representation of the microscopic structure of individual eigenstates. In the following we will briefly present both aspects.

Let us start with a brief discussion of the microscopic aspects of our solutions. Since the exact eigenstates are fully determined by the spectral parameters $(e, \omega, \eta, \gamma)$, the evolution of these parameters in the complex plane allows for tracking structural changes of individual eigenstates as a function of the model parameters. This is demonstrated in Fig. 1 where we show the spectral parameters in the complex plane for three different isospin states and two values of the coupling constant representing weak $g=0.16$ and intermediate $g=0.22$ pairing. As in the $\mathrm{SU}(2) \mathrm{RG}$ model, the expansion of the pair energies $e$ into the complex plane indicates the formation of correlated Cooper pairs [18,19]. Simple counting shows that, for the two $g$ values considered here, the overall number of correlated pairs is, irrespective of isospin, about $6(15 \%)$ and $14(35 \%)$, respectively. The rest of the pairs, still attached to the $s p$ energies, remain almost uncorrelated.

The expansion of the spectral parameters $\omega, \eta$, and $\gamma$ in the complex plane follows the behavior of the pair energies

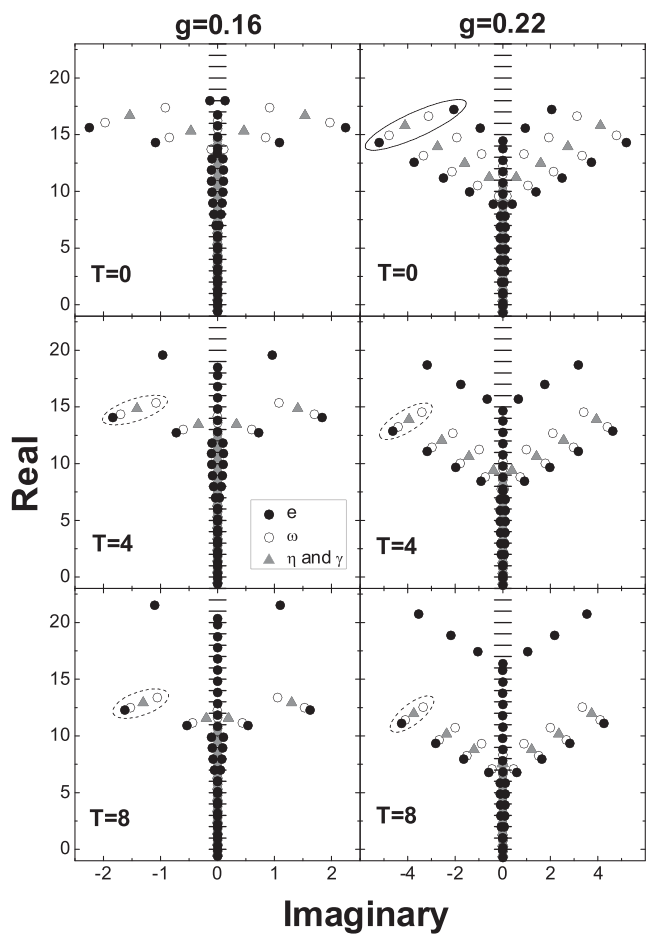

FIG. 1. Complex plane representation of the pair energies $e$ and wave function structure parameters $\omega, \eta$, and $\gamma$, for the lowest-energy states with isospin $T=0,4,8$. The values of $g$ are indicated on the top of the columns. Horizontal lines represent the $s p$ energies $2 \epsilon_{i}=(i-1)$. Clusters representing alpha quartets $(T=0)$ and collective $p$ - $p$ pairs $(T=4$ and $T=8)$ are indicated by solid and dashed ellipses, respectively. $e$; namely, they form parallel arcs to those of the pair energies and they arrange themselves into various clusterlike structures. According to our choice of the LWS an isolated complex parameter $e$ represents a collective $T=1$ $n$ - $n$ Cooper pair; a cluster of one $e$, two $\omega$ 's, one $\eta$, and one $\gamma$ represents a collective $T=1$ Cooper $p$ - $p$ pair, while a cluster of two $e$ 's, two $\omega$ 's, one $\eta$, and one $\gamma$ represents a correlated alphalike quartet. This allows for an unambiguous interpretation of Fig. 1. The existence of correlated quartets is clearly visible in the $T=0$ panel of the figure for strong pairing $g=0.22$. With increasing $T$, these clusters break apart and the net separation of the arcs of the pair energies increases. Moreover, one of the arcs is formed by isolated pair energies $e$ while the other one is constituted by pair energies forming clusters with two $\omega$ 's, one $\eta$, and one $\gamma$ spectral parameters. Physically, it implies a quenching of isoscalar pairing and the formation of two separate conventional $n-n$ and $p$ - $p$ pairing condensates.

Let us turn now to the spectroscopic consequences of the observed microscopic processes along the nuclear symmetry energy (NSE) curve $E(T)$, which can be nicely interpreted in terms of generalized rotations in isospace in the spirit of the isocranking model of Ref. [20]. According to that model the NSE splits into two structurally different even- $T$ and odd- $T$ branches of an isorotational band which can be conveniently parametrized as $E^{(e)}(T)=$ $T(T+\lambda) / 2 J_{T}$ and $E^{(o)}(T)=T(T+\lambda) / 2 J_{T}+\Delta E_{\text {exc }}$, respectively. Here $J_{T}$ stands for the moment of inertia in the isospace (iso-MoI) while $\lambda$ determines the strength of the linear term $\sim T$, which is often called the Wigner energy. The odd- $T$ sequence is shifted up with respect to the even- $T$ branch by a two-quasiparticle [2qp] excitation energy $\Delta E_{\text {exc }}$. Note a beautiful analogy to the spatial collective rotation in even-even nuclei where odd-spin branch is also built upon $2 q p$ excitation.

The calculated inverse of the iso-MoI $\left(1 / J_{T}\right)$ versus $T$, the primary characteristic of the isorotational motion, is shown in Fig. 2(a). Apart from the $\mathrm{SO}(8)$ solution, also two limiting $\mathrm{SO}(5)$ cases invoking only isoscalar and only isovector pairing are depicted [4]. Note that in accordance with the isocranking model (i) all curves converge to the $s p$ splitting $1 / J_{T} \rightarrow \delta \epsilon=1 / 2$ [21], (ii) the $T=1$ paring represents almost perfectly rigid rotation with $1 / J_{T} \approx \delta \epsilon$ irrespectively on $T$ [20], and (iii) the $\mathrm{SO}(8)$ and $T=0$ pairing curves show a characteristic reduction of the $J_{T}$ at low $T$ due to isoscalar pairing collectivity similar to the well recognized reduction of the spatial MoI caused by isovector superfluidity. The increase of $J_{T}$ versus $T$ reflects the disappearance of the isoscalar pairing collectivity caused by fast isorotation which tends to recouple isoscalar (antiparallel coupled isospins) pairs in analogy to the wellknown Coriolis antipairing effect.

The quantity depicted in Fig. 2(b) is directly related to the average difference in pairing correlation energy $E_{\text {corr }} \equiv$ $\langle\hat{H}\rangle-E(T)(\langle\rangle$ denotes the expectation value in the $g=0$ limit) between even- and odd- $T$ branches. This quantity, 


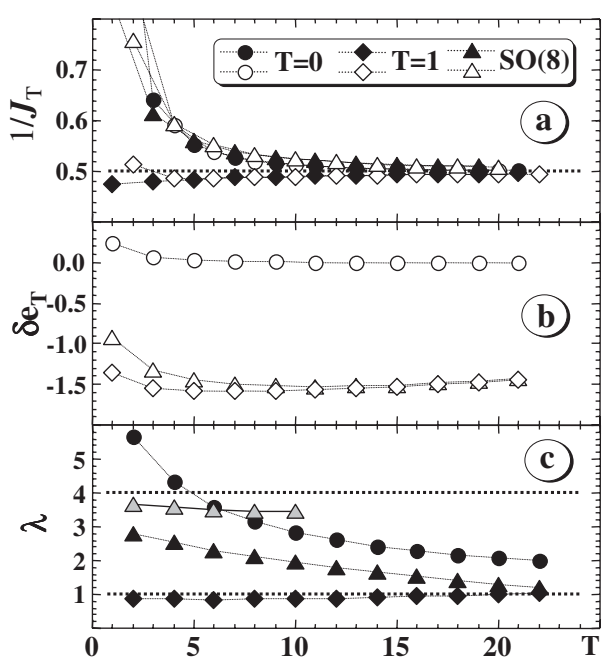

FIG. 2. (a) Inverse of the iso-MoI $\left(1 / J_{T}\right)$, (b) signature splitting $\delta e_{T}$, and (c) linear term enhancement factor $\lambda$ versus $T$ for pure $T=0$ model (circles), pure $T=1$ model (diamonds), and the $\mathrm{SO}(8)$ model (triangles). Solid (open) symbols refer to even(odd-) $T$ branches of $E(T)$. The calculations were done for $g=$ 0.16 except for gray triangles in the lowest panel which mark the $\mathrm{SO}(8)$ solution for $g=0.22$.

known also as the signature splitting, is defined at odd $T$ as $\delta e_{T} \equiv\left[E^{(e)}(T+1)+E^{(e)}(T-1)\right] / 2-E^{(o)}(T)$. At high $T$, where $1 / J_{T} \approx \delta \epsilon$, the signature splitting equals $\delta e_{T}=$ $-\Delta E_{\mathrm{exc}}+\delta \epsilon / 2$ irrespective of $\lambda$. In the case of pure $T=$ 0 pairing building up the isospin proceeds through the isoscalar pair breaking. The correlation energy drops down and the solution goes smoothly over to the $s p$ limit where $\Delta E_{\mathrm{exc}} \rightarrow \delta \epsilon / 2$. Consequently, $\delta e_{T} \rightarrow 0$ as shown in Fig. 2(b). In the $T=1$ pairing case the signature splitting is almost constant and equal $\delta e_{T} \approx-1.5$. In this case we deal with rigid isorotation and odd- and even- $T$ branches are shifted by a constant energy reflecting a difference between correlation energies in odd- (seniority two) and even- $T$ (seniority zero) states ( $2 q p$ energy). Finally, the $\mathrm{SO}(8)$ curve goes smoothly over to the $T=1$ case as the isoscalar pairing disappears with increasing $T$.

Figure 2(c) shows the linear enhancement factor calculated as $\lambda \equiv 2 E_{T}^{(e)} \bar{J}_{T} / T-T$, where $\bar{J}_{T}$ stands for mean value of the iso-MoI. While the $T=1$ pairing yields $\lambda \approx$ 1 , strong enhancement of the Wigner term due to the isoscalar pairing is clearly seen as anticipated [22]. In the $\mathrm{SO}(8)$ case $\lambda$ reaches the Wigner supermultiplet limit $\lambda \sim$ 4 for large $g$ (gray triangles) and drops with decreasing $g$ as well as with increasing $T$ reaching unity for large $T$.

In summary, we have presented the exact solution of the RG model associated to the $\mathrm{SO}(8)$ algebra in the context of nuclear $n$ - $p$ pairing with equal strength for the $T=1$ and $T=0$ interaction components. We have briefly discussed a new technique for solving numerically the Richardson equations which avoids the well-known divergences that have prevented large scale exact solutions. The first application to the nuclear $n$ - $p$ pairing is discussed from both microscopic as well as spectroscopic points of view. In particular, it is shown that the $T=0$ wave function shows alphalike quartet structures that can be recognized by the formation of clusters of spectral parameters containing two pair energies. At high $T$ these alpha clusters dissolve and two separate $p-p$ and $n-n$ superfluid condensates are formed. Spectroscopic consequences of these microscopic processes are discussed and interpreted in terms of generalized rotations in isospace. It is shown that the exact solutions follow nicely the general trends predicted by the isocranking model. The $\mathrm{SO}(8) \mathrm{RG}$ model could play a crucial role in understanding quartet clusterization and quartet condensation in nuclear and cold atom systems.

We acknowledge fruitful discussions with S. Pittel and P. Van Isacker. This work was supported in part by the Spanish MEC under Grant No. FIS2006-12783-C03-01 and by the Polish KBN under Contract No. 1 P03B 05927. S.L.H. acknowledges financial support from Spanish SEUI-MEC. B. E. was supported by the Spanish CE-CAM.

[1] W. Richardson, Phys. Lett. 3, 277 (1963); Phys. Rev. 141, 949 (1966).

[2] Gaudin, J. Phys. (Paris) 37, 1087 (1976).

[3] J. Dukelsky et al., Rev. Mod. Phys. 76, 643 (2004).

[4] J. Dukelsky et al., Phys. Rev. Lett. 96, 072503 (2006).

[5] S. Lerma H. et al., Phys. Rev. C 74, 024314 (2006).

[6] S. Lerma H. and B. Errea, J. Phys. A 40, 4125 (2007).

[7] B. H. Flowers and S. Szpikowski, Proc. Phys. Soc. London 84, 673 (1964).

[8] S. Ch. Pang, Nucl. Phys. A128, 497 (1969).

[9] J. A. Evans et al., Nucl. Phys. A367, 77 (1981).

[10] J. N. Ginnochio, Ann. Phys. (N.Y.) 126, 234 (1980).

[11] P. Lecheminant et al., Phys. Rev. Lett. 95, 240402 (2005); Congjun Wu, Phys. Rev. Lett. 95, 266404 (2005).

[12] D. D. Warner et al., Nature Phys. 2, 311 (2006).

[13] J. A. Maruhn et al., Phys. Rev. C 74, 044311 (2006); M. Chernykh et al., Phys. Rev. Lett. 98, 032501 (2007).

[14] M. Asorey et al., Nucl. Phys. B622, 593 (2002).

[15] A.G. Ushveridze, Quasi-Exactly Solvable Models in Quantum Mechanics (Institute of Physics, Bristol, 1994).

[16] S. Rombouts et al., Phys. Rev. C 69, 061303 (2004).

[17] F. Dominguez et al., J. Phys. A 39, 11349 (2006).

[18] G. G. Dussel et al., Phys. Rev. C 76, 011302(R) (2007).

[19] J. Dukelsky et al., Phys. Rev. Lett. 88, 062501 (2002).

[20] W. Satuła and R. Wyss, Phys. Rev. Lett. 86, 4488 (2001); Phys. Rev. Lett. 87, 052504 (2001).

[21] W. Satuła and R. Wyss, Phys. Lett. B 572, 152 (2003).

[22] J. Engel et al., Phys. Lett. B 389, 211 (1996); W. Satuła and R. Wyss, Phys. Lett. B 393, 1 (1997). 